\title{
MRI IN OSTEOARTHRITIS OF THE HIP: GRADATIONS OF SEVERITY
}

\author{
King C. Li,* Jay Higgs, † Alex M. Aisen,* Kenneth A. Buckwalter, \\ William Martel, * and W. Joseph MCCune $\dagger$ \\ Department of Radiology, ${ }^{*}$ and Department of Rheumatology, $\dagger$ University of Michigan Medical Center, \\ Ann Arbor, Michigan 48109
}

\begin{abstract}
In a prospective study, 10 patients with well-documented osteoarthritis (O.A.) of the hips were imaged using spinecho pulse sequences (TR $=0.5$ to $1.5 \mathrm{~s}$ and $\mathrm{TE}=28$ to $60 \mathrm{~ms}$ ). After analyzing the changes observed, an $\mathrm{MR}$ grading system for assessing severity of $\mathbf{O}$.A. in the hips was developed. Using this grading system and an established grading system for osteoarthritis using roentgenograms (both systems use grades 0-4), two radiologists independently graded the MR studies and plain films separately, twice. The roentgenogram grading system was more accurate in predicting symptoms in the more severe cases, whereas the MR grading system was slightly more useful in the less severe cases. Our results show that $M R$ can demonstrate a spectrum of changes of 0 .A. in the hips. Its ability to directly image articular cartilage makes it a powerful research and clinical tool.
\end{abstract}

Keywords: Hip, MR studies; Hip, osteoarthritis; Magnetic resonance (MR) grading of severity.

\section{INTRODUCTION}

Osteoarthritis or degenerative joint disease is a public health problem of considerable magnitude. In the United States, 30 million people are estimated to have radiologic evidence of the disease. ${ }^{1,2}$ Although the nature of the initial morphologic event in osteoarthritis is debatable, the progressive destruction of articular cartilage and sclerosis of subchondral bone are the basic morphologic changes in the intermediate and late stages of the disease. ${ }^{3}$ With conventional radiography, damage to articular cartilage can only be inferred indirectly by joint space narrowing and subchondral osseous changes. With the advent of magnetic resonance imaging (MRI), intra-articular structures can be directly imaged without using contrast agents or ionizing radiation. ${ }^{4-7}$ Articular cartilage can be well delineated due to its relatively high water content. ${ }^{6}$ Therefore, MRI can potentially be a powerful tool in the evaluation of osteoarthritis. In this study, the authors developed a system, based on MRI, to grade the severity of osteoarthritis in the hips. They assessed the usefulness of this grading system as com- pared to a recognized roentgenographic grading system $^{8}$ by correlating the grades with clinical symptoms and functional limitations of the patients. Finally, the inter- and intra-observer scoring variations in using the two grading systems were also compared.

\section{MATERIALS AND METHODS}

Ten patients with osteoarthritis of the hips, welldocumented clinically and radiologically, were studied prospectively with MRI. There were nine females and one male, with a mean age of 62 (range 33-78). All patients had roentgenograms of the hips within an average of 2.2 months (range $0-4$ months) of the MR examinations. After the MR studies were reviewed, a grading system for evaluation of the severity of O.A. of the hips was developed (see Results), so that the usefulness of MR and roentgenograms could be assessed in an objective manner. Using this system for the MR examinations and a previously developed recognized grading system for the roentgenograms of the hips, (Table 1 ), ${ }^{8}$ two of us, one primarily specializing in MRI, the other with expertise in both MRI
RECEIVED 9/17/87; ACCEPTED 9/30/87.

Acknowledgments - We would like to thank Ms. Nora Fortune for her excellent technical help and Ms. Barbara Smith for typing the manuscript. This study was funded in part by NIH Grant No. 021341 .
Address correspondence and reprint requests to King C. Li, M.D., Department of Radiology, University of Florida College of Medicine, Box J-374, Gainesville, Florida 32610 . 
Table 1. Roentgenograms in O.A. of the Hips Gradations of Severity*

Grade $0=$ Normal

Grade 1 = Possible narrowing of joint space and possible osteophytes around femoral head.

Grade 2 = Definite narrowing of joint space, definite osteophytes and slight sclerosis.

Grade 3 = Marked narrowing of joint space, slight osteophytes, some sclerosis and cyst formation and deformity of femoral head and acetabulum.

Grade 4 = Gross loss of joint space with sclerosis and cysts, marked deformity of femoral head and acetabulum and large osteophytes.

*Adapted and modified from the Council for International Organizations of Medical Sciences, 1963.

and musculoskeletal radiology, working independently of each other, graded the MR studies and roentgenograms separately. Each radiologist graded each examination twice, approximately three weeks apart. The best central coronal MR images were used for grading. The patient identification data on the films were concealed from the radiologists. The functional capacities of the patients were also graded by the attending rheumatologist according to the Steinbrocker, Traeger and Batterman system (Table 2). ${ }^{9}$ For determining inter-observer variations, the MR and roentgenogram scores given by the two observers at each reading were compared using the paired Student $t$ test. For determining intra-observer variations, the MR and roentgenogram scores given by each observer at the first reading were compared to those given by the corresponding observer at the second reading using the paired Student $t$-test.

For correlating MR and roentgenogram scores with the functional capacities of the patients, only the more severely involved hip of each patient was used, that is, the hip with the higher roentgenogram or MR score was used. In the patients with unilateral hip prostheses, the scores of the contralateral hips were used. Since each observer scored each patient twice, four roentgenogram scores and four MR scores were used in each patient. The MR scores of all the patients with the same level of functional capacity were used for calculating an average MR score for that functional capacity category. An average roentgenogram score was similarly calculated for each functional capacity category.

The average MR scores of the different functional capacity categories were compared using the unpaired
Table 2. Classification of Functional Capacity

Class I = Complete functional capacity with ability to carry on all usual duties without handicaps.

Class II = Functional capacity adequate to conduct normal activities despite handicap of discomfort or limited mobility of one or more joints.

Class III = Functional capacity adequate to perform only few or none of the duties of usual occupation or of self care.

Class IV = Largely or wholly incapacitated with patient bedridden or confined to wheel-chair, permitting little or no self care.

Student $t$-test to determine whether there were any statistically significant differences. Similar analysis of the average roentgenogram scores was also carried out.

\section{IMAGING TECHNIQUES}

MRI was performed on a Diasonics MT/S system, based on a $0.35 \mathrm{~T}$ super conducting magnet, using multi-slice, multi-echo pulse sequences. Whole-bodysized imaging coils were used for all patients. The pixel size was about $1.7 \times 1.7 \mathrm{~mm}$. Pulse sequences used included pulse repetition intervals $(T R)=0.5 \mathrm{~s}$, echo delay time $(\mathrm{TE})=28-30 \mathrm{~ms}$ ( 5 patients); TR $=$ $1.0 \mathrm{~s}, \mathrm{TE}=28-30 \mathrm{~ms}(9$ patients $) ; \mathrm{TR}=1.0 \mathrm{~s}, \mathrm{TE}=$ $56-60 \mathrm{~ms}$ (five patients); $\mathrm{TR}=1.5 \mathrm{~s}, \mathrm{TE}=30-40 \mathrm{~ms}$ (two patients). All patients had axial and coronal scans. Contiguous $10 \mathrm{~mm}$ thick sections were obtained in 8 patients and $5 \mathrm{~mm}$ thick contiguous sections were used in two patients.

\section{RESULTS}

Of the 10 patients studied, two had unilaterai total hip prostheses, therefore 18 hips were included in this study. Of the pulse sequences used, a pulse repetition interval (TR) of 1.0 second and an echo delay time (TE) of 30 to $40 \mathrm{~ms}$ were found to be the most useful. With this pulse sequence, sufficient anatomical details could be obtained while maintaining enough $T_{1}$ contrast for detecting abnormalities in the bone marrow (Fig. 1). Coronal images were found to be more useful than axial images. The newly developed MR grading system for the severity of O.A. in the hips is shown in Table 3. The MR grading system was developed such as to incorporate all the MR changes observed in the osteoarthritic hips studied and could be easily compared to the roentgenographic grading sys- 


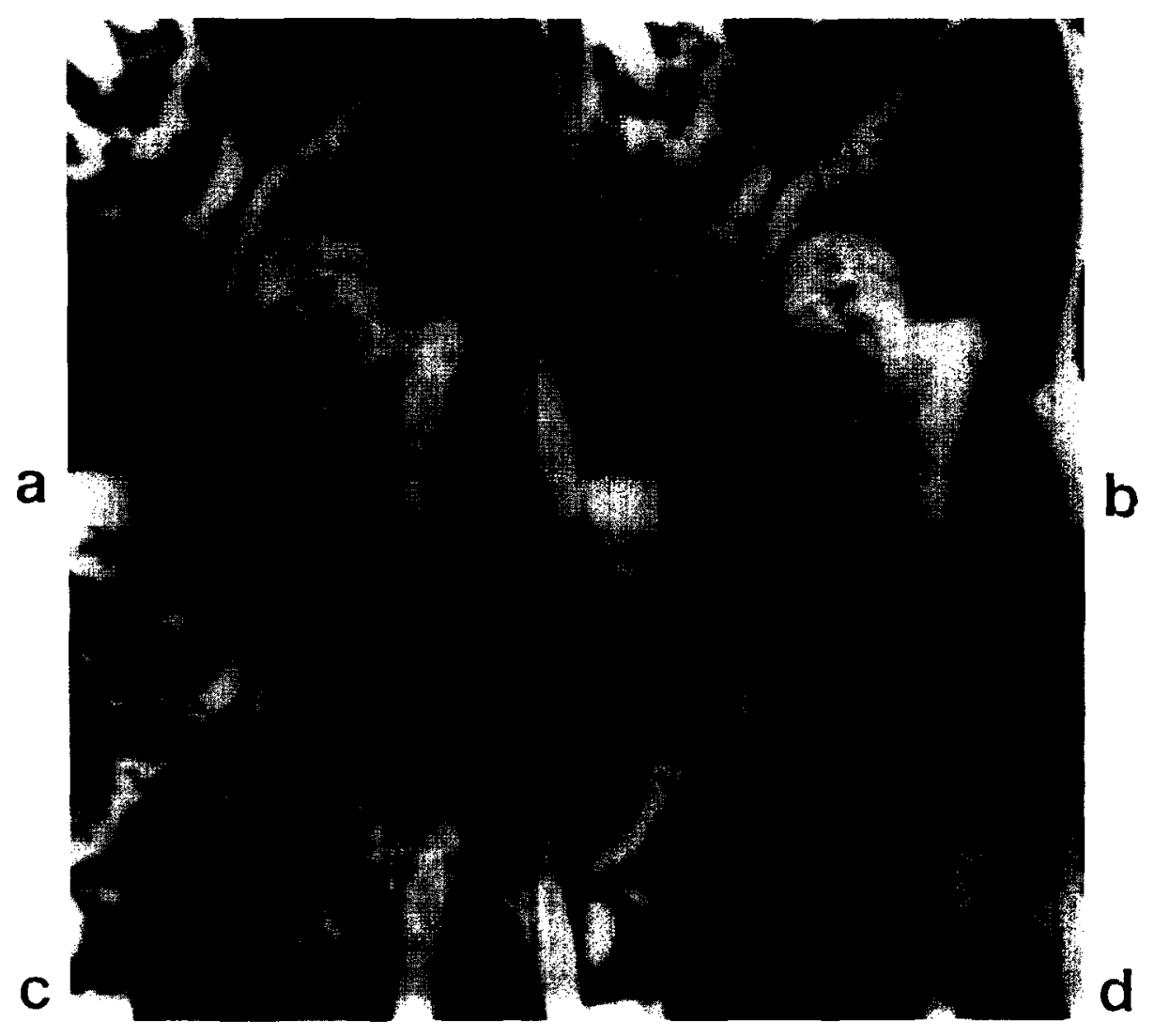

Fig. 1. Coronal image of the left hip of an asymptomatic normal volunteer, (a) at $\mathrm{TR}=0.5 \mathrm{sec}, \mathrm{TE}=40 \mathrm{msec}$, good contrast between red and yellow marrow is seen in the proximal femur but the articular cartilage (arrows) is not optimally seen, (b) at $T R=1.0 \mathrm{sec}, T E=40 \mathrm{msec}$, the articular cartilage (arrows) is better defined and the contrast between red and yellow marrow is still good, (c) at $\mathrm{TR}=1.5 \mathrm{sec}, \mathrm{TE}=\mathbf{4 0} \mathrm{msec}$, not much contrast is seen between red and yellow marrow, (d) at $\mathrm{TR}=1.5 \mathrm{sec}, \mathrm{TE}=80 \mathrm{msec}$, the anatomical detail and contrast between red and yellow marrow are poor.

Table 3. MR in O.A. of the Hips-Gradations of Severity

Grade $0=$ Normal

Grade 1 = Nonhomogeniety of high signal in articular cartilage.

Grade 2 = Nonhomogeniety and discontinuity of high signal in articular cartilage, blurring of trabecular pattern and overall loss of signal in the femoral head and neck.

Grade 3 = Loss of high signal of articular cartilage, blurring of trabecular pattern and overall loss of signal in the femoral head and neck, irregularities of the cortical outlines of femoral head and acetabulum, grey zone between acetabulum and femoral head and regions of intermediate signal intensity outlined by dark rims in the femoral head.

Grade $4=$ Changes of grade 3 plus more decrease in signal and deformity of the femoral head. tem shown in Table 1. Examples illustrating the typical MR changes seen in osteoarthritis of the hips are shown in Figs. 2-6. The examples are chosen so as to correspond to each of the grades used in the MR scoring system. The corresponding roentgenograms are also shown for comparisons.

Of the 10 patients, there were 3,5 , and 2 patients in the Grade IV, III, and II functional capacity categories respectively. Of the three patients in the Grade IV functional capacity category, two had symmetric symptoms and one was more symptomatic on the left side. In the patients with symmetric symptoms, the roentgenogram grades were equal on both sides whereas MR grades were asymmetric. In the patient with asymmetric symptoms, both MR and roentgenogram grades were higher on the more symptomatic side.

Of the 5 patients in the Grade III functional capacity category, 2 had unilateral total hip prostheses, 2 had asymmetric symptoms and 1 had symmetric symp- 


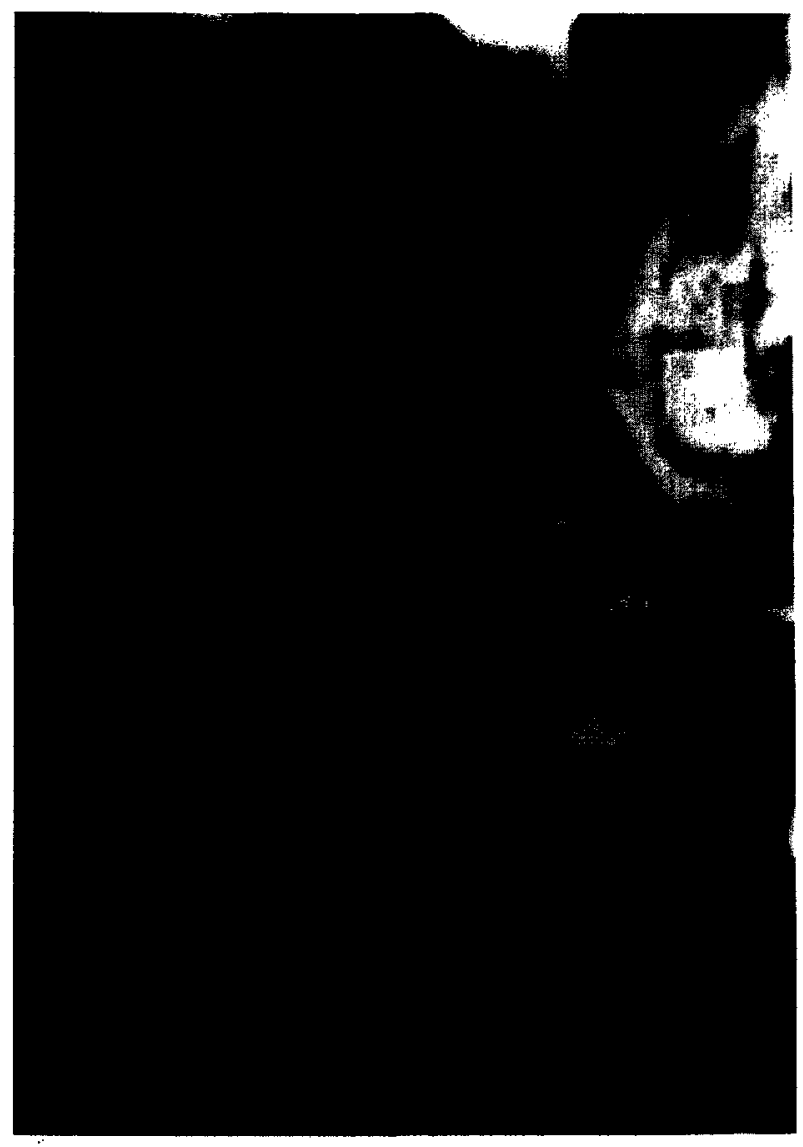

Fig. 2. Coronal $M R$ image at $T R=1.0 \mathrm{sec}, T E=40 \mathrm{msec}$ of the right hip of a 26-year-old female asymptomatic normal volunteer. Notice the homogeneous high signal of the articular cartilage (arrows) and the well-defined trabecular pattern in the femoral head and neck (arrowhead).

toms. In the patients with asymmetric symptoms, both MR and roentgenogram grades were higher on the symptomatic sides. In the patient with symmetric symptoms, both MR and roentgenogram grades were equal on both sides.

Both patients in the Grade II functional capacity category had asymmetric symptoms. In one patient, both MR and roentgenogram grades were higher on the symptomatic side. In the other patient, MR grades were higher on the more symptomatic side whereas roentgenogram grades were equal on both sides.

The average roentgenogram scores of the different functional capacity categories were $4.00(N=12$, $\mathrm{SD}=0)$ for Grade IV, $3.65(N=20, \mathrm{SD}=0.49)$ for Grade III and $2.88(N=8, \mathrm{SD}=0.35)$ for Grade II respectively. The differences between the average roentgenogram scores of the different functional capacity categories were statistically significant $(p<$
0.01 for Grade IV vs Grade III and $p<0.001$ for Grade III vs Grade II).

The average MR scores were $3.92(N=12, \mathrm{SD}=$ $0.29)$ for Grade IV, $3.65(N=20, \mathrm{SD}=0.67)$ for Grade III and $2.50(N=8, \mathrm{SD}=0.93)$ for Grade II. The average MR scores of functional capacity categories Grade IV and Grade III were not significantly different statistically $(p>0.1)$, but the difference between the average MR scores of Grade III and Grade II was statistically significant $(p<0.01)$.

At both first and second readings, the roentgenogram scores given by the two observers were identical in 14 hips and differed by 1 grade in 4 hips. The interobserver differences were statistically significant $(p<$ 0.05 ). The roentgenogram scores given by observer 1 at the two readings were identical in 16 hips and differed by 1 grade in 2 hips ( $p>0.1$ ). The roentgenogram scores given by observer 2 at the two readings were identical in 14 hips and differed by 1 grade in 4 hips $(p<0.05)$.

At the first reading, the MR scores given by the two observers were identical in 9 hips and differed by 1 grade in 9 hips $(p<0.01)$. At the second reading, the MR scores of the 2 observers were identical in 12 hips, differed by 1 grade in 5 hips and differed by 2 grades in 1 hip $(p<0.01)$. The MR scores given by observer 1 at the two readings were identical in 15 hips and differed by 1 grade in 3 hips $(p<0.05)$. The MR scores given by observer 2 at the two readings were identical in 9 hips, differed by 1 grade in 7 hips and differed by 2 grades in 2 hips ( $p<0.002)$.

\section{DISCUSSION}

Although osteoarthritis is the commonest form of joint disease, its pathogenesis remains poorly defined. Most observers believe that local cartilage degeneration, including fibrillation of the cartilage surface, and formation of cartilage microfractures and erosions, are the primary lesions in osteoarthritis, and that these changes lead to secondary bone changes including sclerosis of subchondral bone, trabecular microfractures and increased density of trabecular bone in the subchondral region, and cyst formation. ${ }^{10,11}$ These changes are accompanied by increased water content of cartilage, altered quantity and quality of proteoglycans and possible in vivo synthesis of Type I, in addition to Type II, collagen. ${ }^{12,13}$ The specific effect of each of these changes on the signal intensity of articular cartilage will require further investigation. Additional changes may be caused by replacement of articular cartilage with fibrocartilage, which may cause decrease in signal intensity. Thus, alterations of articular 


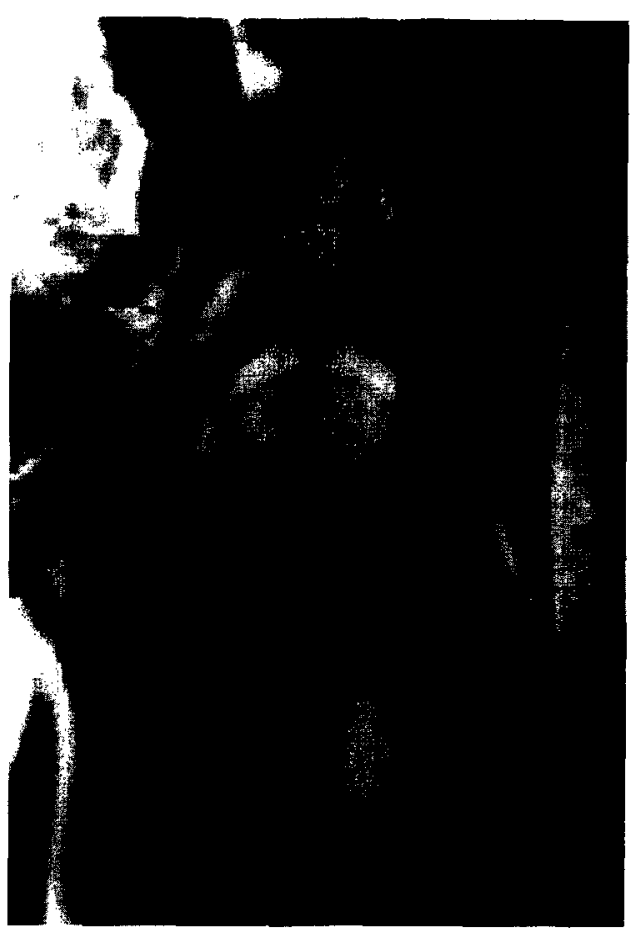

A

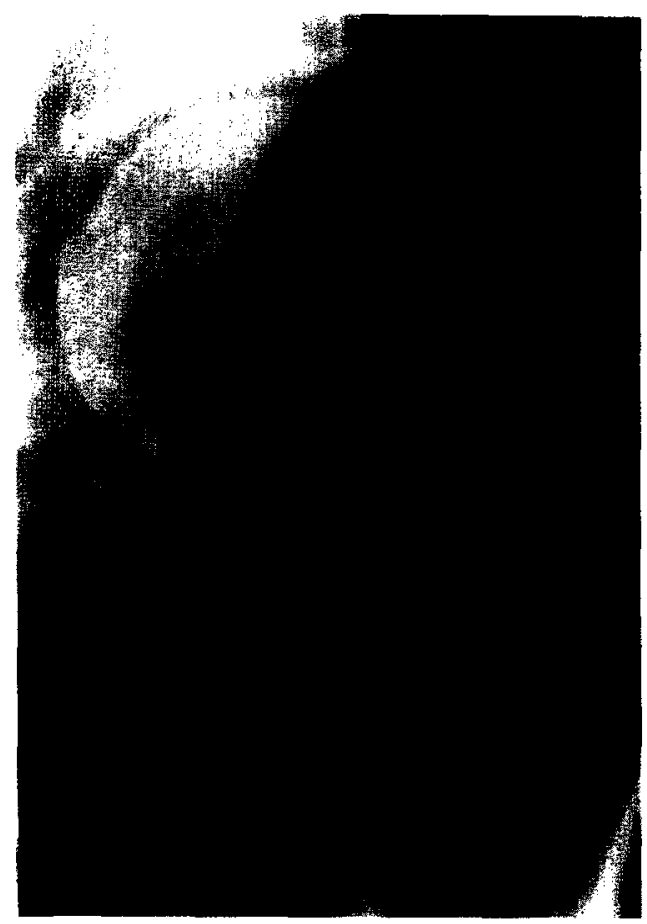

B

Fig. 3. (A) Coronal MR image at TR $=1.0 \mathrm{sec}, \mathrm{TE}=30 \mathrm{msec}$ of a left hip with characteristic changes of grade 1 osteoarthritis (O.A.). Notice the inhomogeniety of the high signal of articular cartilage (arrows). The trabecular pattern of the fernoral head and neck is fairly well defined (arrowhead). (B) Corresponding roentgenogram shows no definite loss of joint space or other significant abnormality

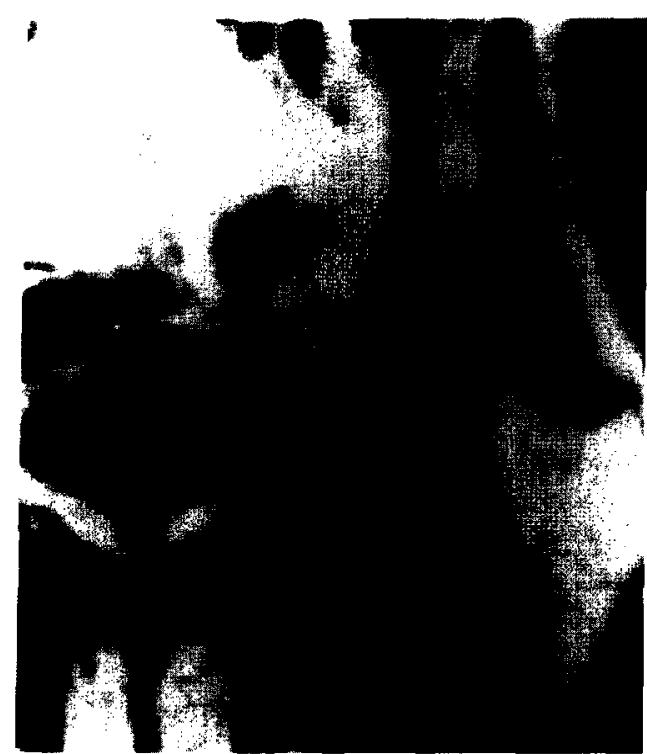

A

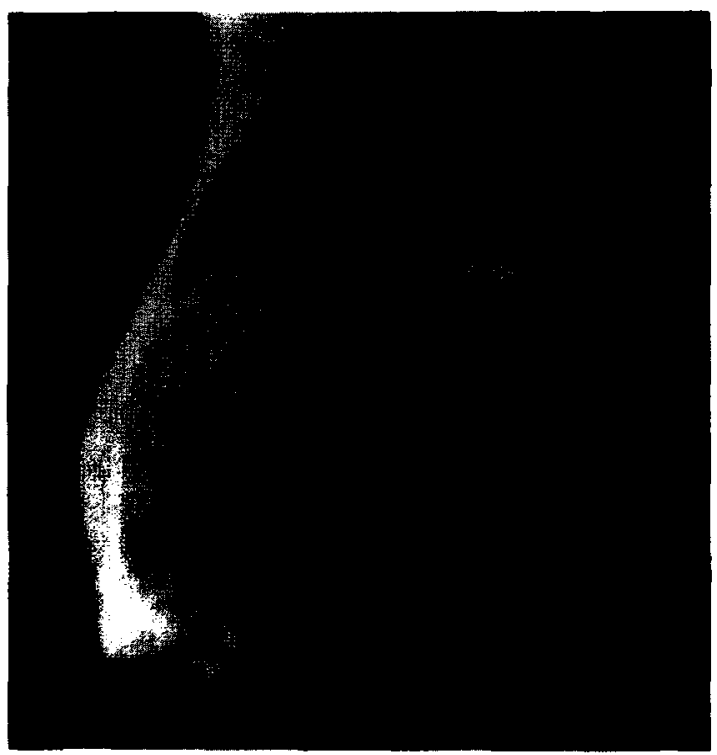

B

Fig. 4. (A) Coronal MR image at $T R=1.0 \mathrm{sec}, T E=28 \mathrm{msec}$, of a left hip with characteristic changes of grade 2 O.A. Notice the discontinuity of the high signal of articular cartilage (arrows). Lateral osteophytes are also seen (small arrows). (B) Corresponding roentgenogram shows definite loss of joint space superiorly (arrow), lateral osteophytosis (small arrows) and sclerotic changes of subchondral bone (arrowhead). 


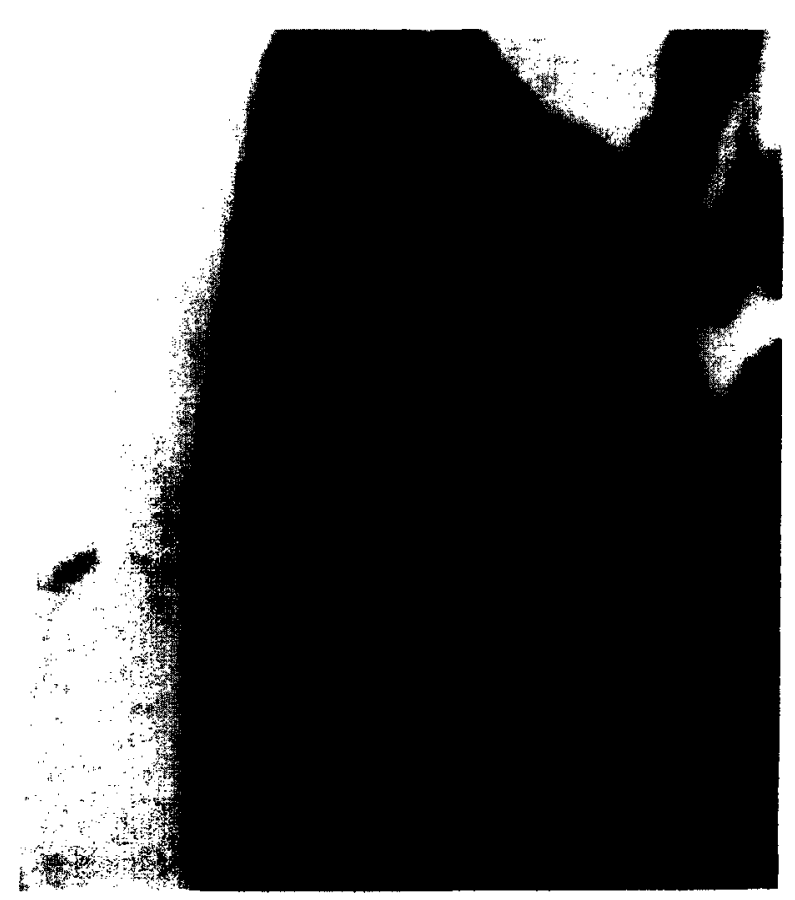

A

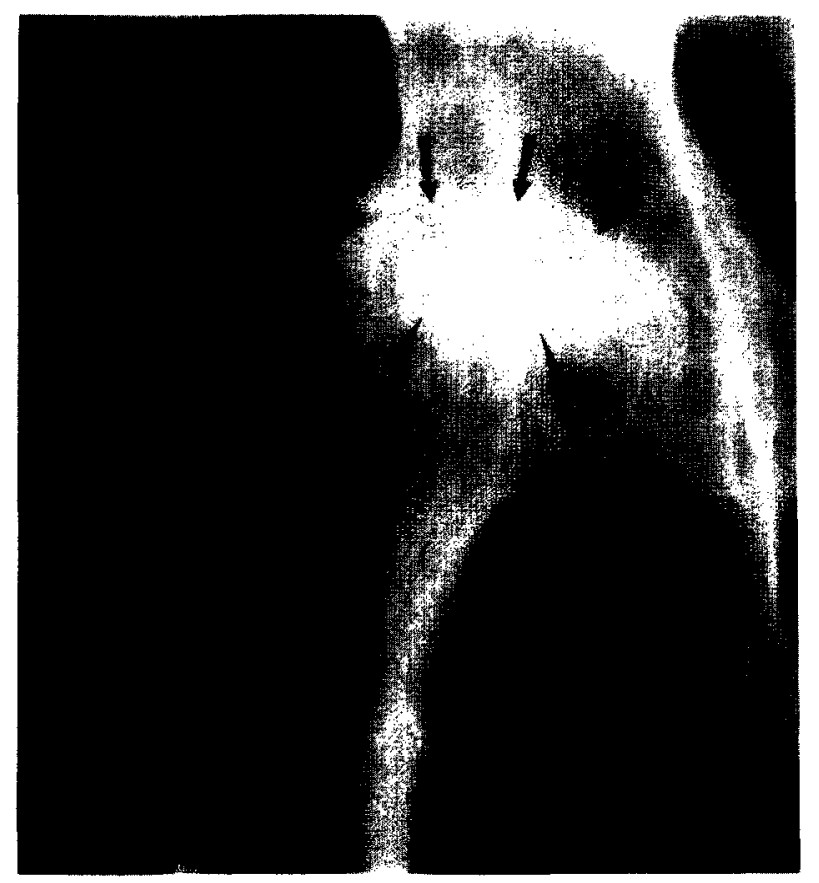

B

Fig. 5. (A) Coronal MR image at $T R=1.0 \mathrm{sec}, T E=30 \mathrm{msec}$ of a right hip with characteristic changes of grade 3 O.A. Notice the total loss of the high signal of articular cartilage and a grey zone between the acetabulum and the femoral head (small arrows). There is a region of intermediate intensity outlined by a dark rim in the superior portion of the femoral head (arrows) which most likely represents a subchondral cyst. The overall signal of the femoral head is decreased and the major trabeculae of the femoral head and neck is scen as a broad dark band (arrowhead). (B) Corresponding plain film shows gross loss of joint space (arrows) with marked sclerosis of the subchondral bone (arrowheads). Lateral osteophytosis is also seen. The large subchondral cyst in the superior portion of the femoral head is not as well visualized as in the MR image.

cartilage in osteoarthritis potentially demonstrable by MR may include not only morphologic changes related to cartilage narrowing but also signal intensity changes due to biochemical alterations, the net effect of which is still unknown.

Since the MR images were not correlated directly with pathologic changes in this study, we can only speculate on the pathologic bases of the MR findings. The observed nonhomogeneity and discontinuity of high signal in articular cartilage in early O.A. may be due to the decrease in its physical thickness and/or biochemical changes. The blurring of trabecular pattern and overall loss of signal in the femoral head and neck in the intermediate and late stages of O.A. may be the MR counterpart of sclerotic changes seen in roentgenograms. The irregularities of cortical outlines of femoral head and acetabulum seen in the MR images of hips with late O.A. may be caused by small subchondral cysts. The larger subchondral cysts may be the morphologic correlates of the regions of intermediate signal intensities outlined by dark rims seen in MR images. Finally, we speculate that the grey zone between the femoral head and acetabulum seen in the later stages of O.A. may be caused by signal averaging between the bone marrow fat, the markedly sclerotic trabecular bone and the reparative coat of fibrocartilage and articular cartilage that may be present.

It is apparent from our study that MR can demonstrate a spectrum of O.A. changes in the hip, the grading of which may in the future facilitate the reporting of these findings and correlations with clinical symptomatology. In our study, the newly developed MR grading system was less accurate, as compared to the roentgenogram grading system, in predicting symptoms in the more severe cases. However, in the less severe cases, the MR grading system was slightly more accurate in predicting symptoms. This may be related to the ability of MR in directly imaging the articular cartilage, which may be useful in assessing the effectiveness of different treatments and working out the pathogenesis of O.A. ${ }^{14-16}$

The average roentgenogram scores of the different functional capacity categories were significantly different statistically in our study, indicating that the higher roentgenogram grades were generally asso- 


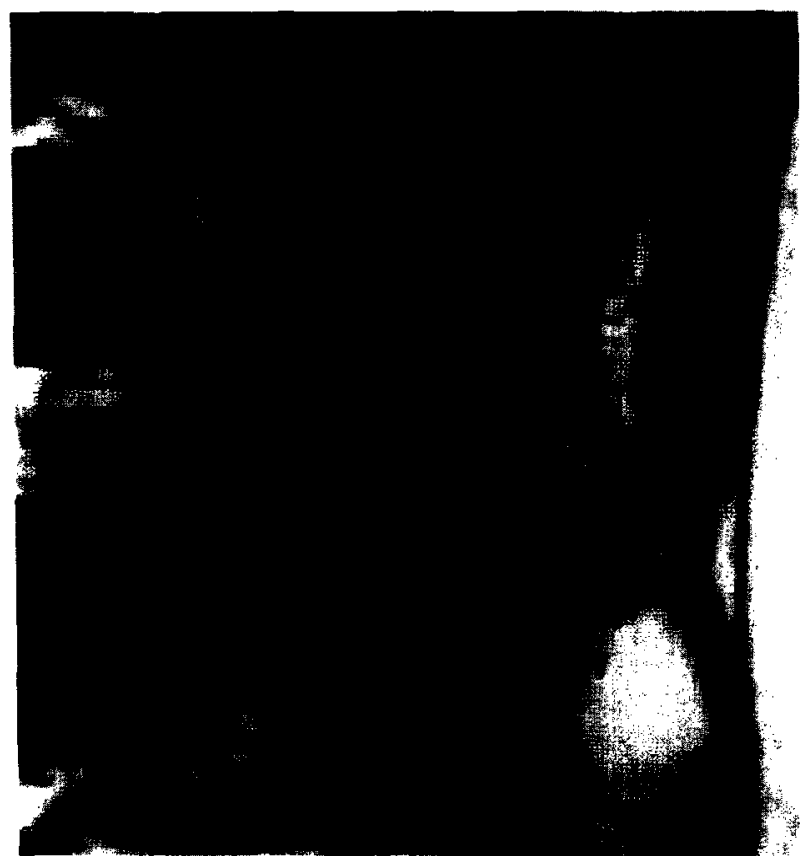

Fig. 6A. Coronal MR image at $\mathrm{TR}=1.5 \mathrm{sec}, \mathrm{TE}=30 \mathrm{msec}$ of a left hip with characteristic changes of grade 4 O.A. Notice the total loss of the articular cartilage and the grey zone between the acetabulum and femoral head (arrows). The cortical outlines are very irregular (small arrows) and the overall shape of the femoral head is grossly deformed. There is also a diffuse loss of signal in the entire femoral head and the medial side of the femoral neck (arrowhead).

ciated with more significant functional limitations. Although the correlation of MR grades and functional capacities was good in the less severe cases, the correlation was poor in the more severe cases. This was at least in part due to the lack of experience of the observers in using the MR grading system, as suggested by the high inter- and intra-observer variations of the MR scores. This may also be related to the relative inability of the observers to differentiate clearly the features on which the criteria are based for classification. This may in turn be related to the relatively inferior spatial resolution of the MR scans. With higher strength magnets and surface coil techniques the pixel size and slice thickness may be substantially decreased and better results with MR can be expected. Another factor to consider is that the features chosen for differentiating Grade III and Grade IV in the MR grading scheme may be inappropriate, and modifications may be required. Further experience with more patients and improved techniques will be needed before any definitive statements can be made regarding the usefulness of MR in predicting symptoms and functional limitations of patients with severe O.A.

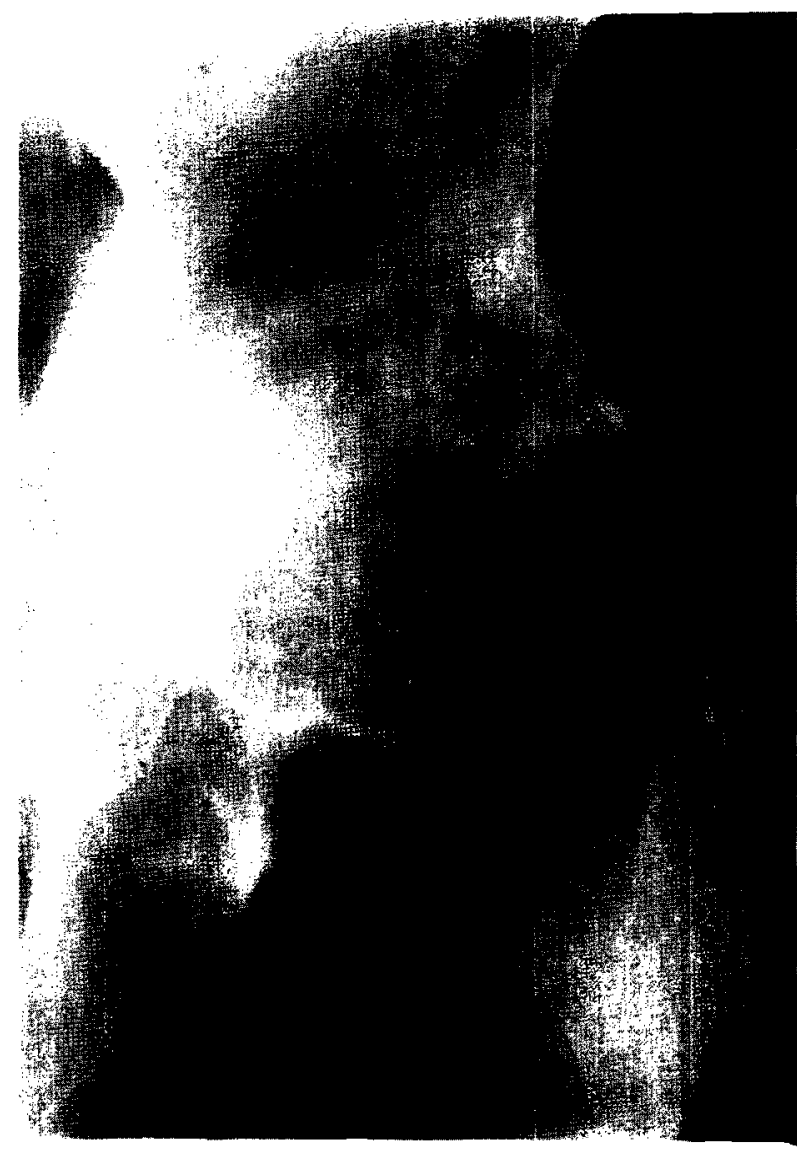

Fig. 6B. Corresponding plain film shows gross loss of joint space with marked sclerosis of the subchondral bones. Marked deformity of the femoral head and acetabulum with osteophytosis is also seen. There is also a subchondral cyst in the superior portion of the femoral head (arrow) which was also noted in an MR image $10 \mathrm{~mm}$ posterior to the image shown in (A).

In conclusion, our study shows that MR can demonstrate a spectrum of changes of O.A. in the hips. There is a possibility that MR may correlate better with symptoms in the less severe cases as compared to roentgenograms. Although the exact role of MR as a research and/or clinical tool in O.A. is still to be defined, we believe that its ability in directly imaging articular cartilage makes it a powerful addition to current diagnostic methods.

\section{REFERENCES}

1. Hadler, N.W. Osteoarthritis as a public health problem. Clin. Rheum. Dis. 11:175; 1985.

2. Rouff, G.E. The pain of osteoarthritis. Am. J. Med. (Suppl 3A):96; 1986.

3. Meachim, G.; Brooke, G. The pathology of osteoarthritis. Moskowitz, R.W.; Howell, D.S.; Goldberg, V.M.; 
Mankin, H.J., eds. Osteoarthritis - diagnosis and management. Philadelphia, PA: W. B. Saunders; 1984: 29-42.

4. Beltran, J.; Noto, A.M.; Mosure, J.C.; Weiss, K.L.; Zuelzer, W.; Christoforidis, A.J. The knee: surface coil MR imaging at 1.5T. Radiology. 159:747-751; 1986.

5. Huber, D.J.; Sauter, R.; Mueller, E.; Requardt, M.; Weber, H. MR imaging of the normal shoulder. Radiology. 158:405-408; 1986.

6. Li, K.C.; Henkelman, R.M.; Poon, P.Y.; Rubenstein, J. MR imaging of the normal knee. J. Comput. Assist. Tomogr. 6:1147-1154; 1984.

7. Littrup, P.J.; Aisen, A.M.; Braunstein, E.M.; Martel, W. Magnetic resonance imaging of femoral head development in roentgenographically normal patients. Skeletal. Radiol. 14:159-163; 1985.

8. Council for International Organizations of Medical Sciences. The epidemiology of chronic rheumatism, Volume 2, Atlas of Standard Radiographs of Arthritis. Oxford-Blackwell; 1963: 44.

9. Steinbrocker, O.; Traeger, C.H.; Batterman, R.C. Therapeutic criteria in rheumatoid arthritis. JAMA 140:659-662; 1949.
10. Cooke, T.V.E. Pathogenetic mechanism in polyarticular ostenarthritis. Clin. Rheum. Dis. 11:203-237; 1985.

11. Dieppe, P. Osteoarthritis: are we asking the wrong questions? Br. J. Rheumatol. 23:161-165; 1985.

12. Fell, H.B.; Jubb, R.W. The effect of synovial tissue on the breakdown of articular cartilage in organ culture. Arthritis. Rheum. 20:1359-1371; 1977.

13. Mankin, H.J.; Brandt, K.D. Biochemistry and metabolism of cartilage in osteoarthritis. In: Moskowitz, R.W.; Howell, D.S.; Goldberg, V.M.; Mankin, H.J., eds. Osteoarthritis - diagnosis and management. Philadelphia, PA: W.B. Saunders, 1984: 43-79.

14. Huskisson, E.C.; Doyle, O.V.; Lanham, J.G. Drug treatment of osteoarthritis. Clin. Rheum. Dis. 11:421$431 ; 1985$.

15. Norman, A. Roentgenologic diagnosis. Moskowitz, R.W.; Howell, D.S.; Goldberg, V.M.; Mankin, H.J., eds. Osteoarthritis - diagnosis and management. Philadelphia, PA: W.B. Saunders; 1984: 155-184.

16. Resnick, D.; Niwayama, G. Degenerative disease of extraspinal locations. In: Resnick, D.; Niwayama, G., eds. Diagnosis of bone and joint disorders. Philadelphia, PA: W.B. Saunders; 1981: 1270-1367. 